\title{
IUPAC International Congress for Analytical Sciences 2011 (ICAS2011)
}

\author{
Kyoto International Conference Center, Kyoto, Japan, May 22-26, 2011
}

(C) Springer-Verlag 2010

ICAS2011, an international congress for analytical sciences coorganized by The Japan Society for Analytical Chemistry and International Union of Pure and Applied Chemistry will be held in Kyoto, Japan, from May 22nd to 26th, 2011. Approximately 1,000 participants from about 50 countries are expected to attend.

\section{Scientific Program:}

The scientific program consists of plenary, keynote, and invited lectures by distinguished scientists and contributed oral and poster presentations.

Symposia organized will include the following topics:

- Advanced instrumental analysis

Chromatography and electrophoresis

Molecular spectroscopy

Atomic spectroscopy

Electrochemistry

Microanalysis and microfluidics

Mass spectrometry

Sensor systems

Novel separation systems

$\mathrm{X}$-ray analysis

- Nanoscience and nanotechnology

Nanoparticles and nanomaterials

Microparticle and interface analysis

- Biological and bioanalysis

Bioanalysis

Biomolecular imaging

Omics

Pharmaceutical science

- Environmental science

Environmental analysis

Geochemical analysis
- Safety, security, and sustainability

Food science

Forensics and homeland security

- Special session

JST special session

Plenary lectures by world's leading scientists are as follows:

- Yoshinobu Baba, Nagoya University

- Takashi Kakiuchi, Kyoto University

- Chad Mirkin, Northwestern University

- Royce Murray, University of North Carolina at Chapel Hill

- Alfredo Sanz-Medel, University of Oviedo

\section{Exhibition:}

Exhibition of new analytical instruments and resources will be scheduled.

Key dates:

September 30th, 2010 Abstract Deadline for Oral and Poster Presentation

December 17th, 2010 Second Circular and Preliminary Program

February 28th, $2011 \quad$ Deadline for Early Registration

May 22nd, $2011 \quad$ Opening of the Congress

\section{Information:}

For abstract submission, program, registration, accommodation, exhibition, and transportation details, visit the conference web site at http://www.icas2011.com/ or contact Koji Otsuka, Secretary General, Graduate School of Engineering, Kyoto University, Katsura, Nishikyo-ku, Kyoto, 615-8510, Japan, E-mail: ICAS2011_secretary@anchem.mc.kyoto-u.ac.jp, Fax: +81-75-383-2450. 